\title{
ARTICLE OPEN Global profiling of O-GlcNAcylated and/or phosphorylated proteins in hepatoblastoma
}

\author{
Hang Song ${ }^{1}$, Ji Ma ${ }^{1}$, Zhixuan Bian ${ }^{1}$, Shuhua Chen ${ }^{2}$, Jiabei Zhu ${ }^{1}$, Jing Wang ${ }^{3}$, Nan Huang ${ }^{4}$, Minzhi Yin $^{5}$, Fenyong Sun ${ }^{4}$, Min $\mathrm{Xu}^{3}$ and
} Qiuhui Pan ${ }^{1}$

O-linked- $\beta$-N-acetylglucosamine (O-GIcNAc) glycosylation (O-GlcNAcylation) and phosphorylation are critical posttranslational modifications that are involved in regulating the functions of proteins involved in tumorigenesis and the development of various solid tumors. However, a detailed characterization of the patterns of these modifications at the peptide or protein level in hepatoblastoma $(\mathrm{HB})$, a highly malignant primary hepatic tumor with an extremely low incidence in children, has not been performed. Here, we examined O-GICNAc-modified or phospho-modified peptides and proteins in HB through quantitative proteomic analysis of HB tissues and paired normal liver tissues. Our results identified 114 O-GlcNAcylated peptides belonging to 78 proteins and 3494 phosphorylated peptides in 2088 proteins. Interestingly, 41 proteins were modified by both O-GlcNAcylation and phosphorylation. These proteins are involved in multiple molecular and cellular processes, including chromatin remodeling, transcription, translation, transportation, and organelle organization. In addition, we verified the accuracy of the proteomics results and found a competitive inhibitory effect between O-GIcNAcylation and phosphorylation of HSPB1. Further, O-GlcNAcylation modification of HSPB1 promoted proliferation and enhanced the chemotherapeutic resistance of HB cell lines in vitro. Collectively, our research suggests that O-GICNAC-modified and/or phospho-modified proteins may play a crucial role in the pathogenesis of HB.

Signal Transduction and Targeted Therapy (2019)4:40; https://doi.org/10.1038/s41392-019-0067-4

\section{INTRODUCTION}

Hepatoblastoma (HB), which originates from the abnormal development of pluripotent stem cells or hepatic progenitor cells, is a highly malignant embryonic hepatocellular carcinoma in children. According to the National Cancer Institute, the occurrence of $\mathrm{HB}$ is extremely rare, with approximately 1.5 cases per million individuals each year. ${ }^{1}$ Despite the improvements and advancements in surgical management, medication, and postoperative chemoradiotherapy for $\mathrm{HB}$, the majority of $\mathrm{HB}$ patients still have an unfavorable prognosis with a high risk of early death due to difficulties in diagnosis and the high frequency of metastasis and recurrence. ${ }^{2,3}$ Therefore, it is critical to fully understand the molecular mechanism and pathogenesis of $\mathrm{HB}$, which would be helpful to improve the early diagnosis, treatment, and prognosis of HB patients.

Posttranslational modifications (PTMs) of proteins are important mechanisms for modulating protein function and increasing the proteome complexity in organisms. ${ }^{4}$ O-linked- $\beta$-N-acetylglucosamine (O-GlcNAc) glycosylation (O-GlcNAcylation) involves a single $\mathrm{N}$-acetylglucosamine molecule being transferred to or removed from serine (Ser) and/or threonine (Thr) residues of proteins by O-GlcNAc transferase (OGT) or O-GlcNAcase, respectively. ${ }^{5}$ O-GIcNAcylation is a widespread, dynamic and reversible PTM that is involved in the regulation of diverse cellular regulatory processes, including protein-protein interactions, cellular signal transduction, epigenetic reprogramming, and metabolic regulation. ${ }^{6-8}$ Moreover, numerous O-GIcNAcylated proteins have been found to be associated with the progression and development of various kinds of cancers. For example, O-GlcNAcylation of the ribosomal protein RACK1 at Ser122 resulted in a dramatic decrease in the malignancy of hepatocellular carcinoma cells in vitro and in vivo. ${ }^{9}$

Phosphorylation of proteins by protein kinases has been demonstrated to play a regulatory role in many biological functions, such as signal transduction, the cell cycle, cell metabolism, and protein subcellular localization. ${ }^{10-13}$ For instance, a previous study showed that p-38 mitogen-activated protein kinases (MAPKs), extracellular signal-related kinase (ERK 1/2), and Akt were phosphorylated and involved in apoptosis and cell cycle arrest in HB cells. ${ }^{14}$ Interestingly, Ser/Thr sites in proteins can be phosphorylated, as well as O-GlcNAcylated, suggesting the possibility of the dynamic and extensive crosstalk between O-GlcNAcylation and phosphorylation. ${ }^{15}$ Indeed, accumulating evidence has shown that the majority of phosphorylated proteins are also O-GIcNAcylated. ${ }^{16,17}$ Further, an interaction between O-GIcNAcylation and phosphorylation was demonstrated in the mouse synaptosome and in the Arabidopsis model plant system. ${ }^{18,19}$ Previously, we elucidated that global O-GlcNAcylation expression levels were increased in liver cancer cell

\footnotetext{
${ }^{1}$ Department of Laboratory Medicine, Shanghai Children's Medical Center, School of Medicine, Shanghai Jiaotong University, 200127 Shanghai, China; ${ }^{2}$ Department of Laboratory Medicine, Yunfu People's Hospital, 527300 Guangdong, China; ${ }^{3}$ Department of Surgery, Shanghai Children's Medical Center, School of Medicine, Shanghai Jiaotong

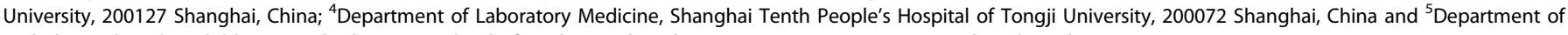
Pathology, Shanghai Children's Medical Center, School of Medicine, Shanghai Jiaotong University, 200127 Shanghai, China

Correspondence: Min Xu (jackxm1236@126.com) or Qiuhui Pan (panqiuhui@scmc.com.cn)

These authors contributed equally: Hang Song, Ji Ma, Zhixuan Bian
}

Received: 3 March 2019 Revised: 28 May 2019 Accepted: 27 June 2019

Published online: 11 October 2019 

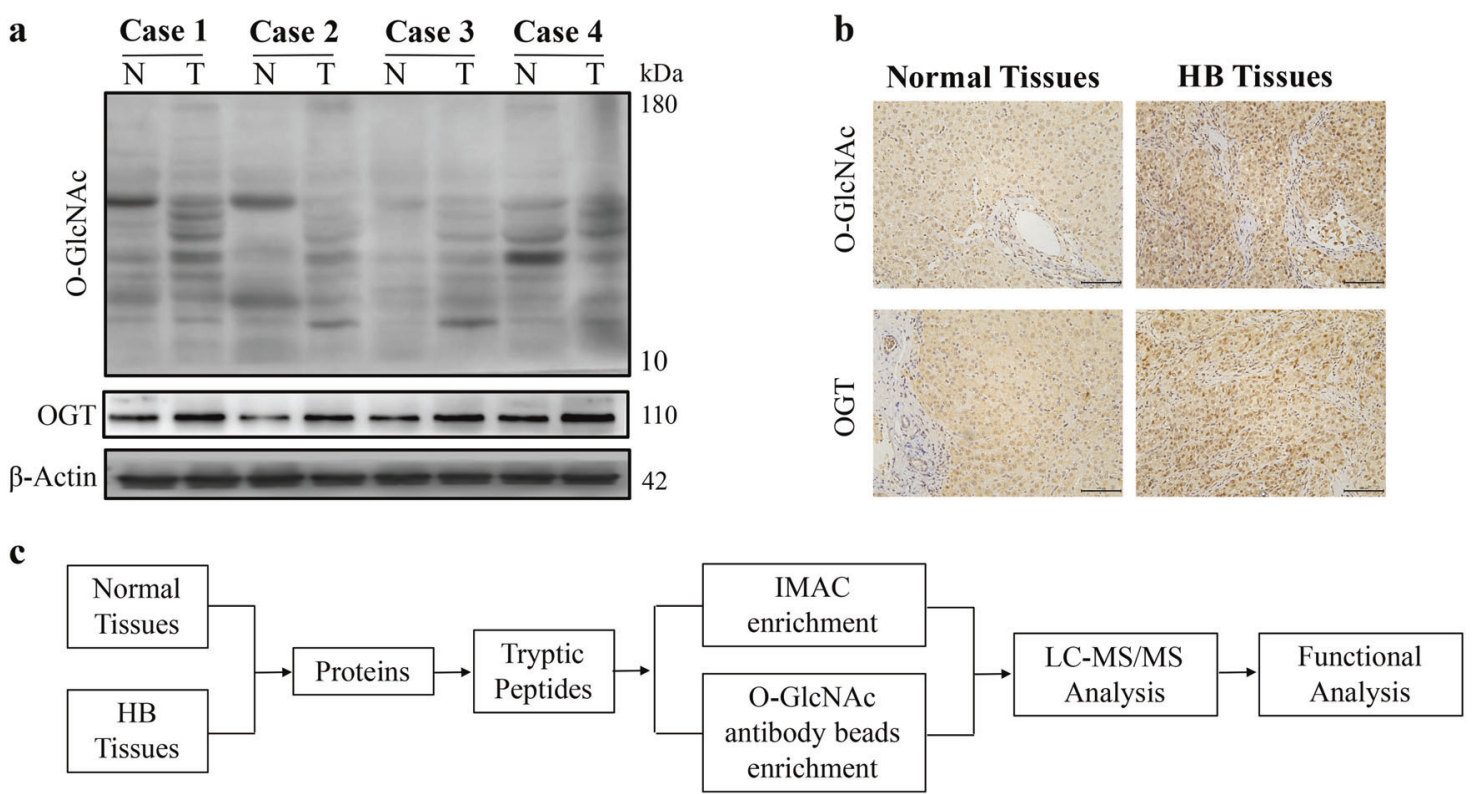

Fig. 1 O-GlcNAcylation and phosphorylation in HB tissues. The expression levels of total O-GlcNAcylation and OGT in HB tissues and normal tissues were measured by using western blot (a) and immunohistochemical analyses (b, scale bars, $100 \mu \mathrm{m})$. c Flowchart for the enrichment and identification of O-GlcNAcylated and phosphorylated peptides by proteomic analysis. All experiments were repeated three times

lines and tissues, which simulated the tumorigenesis of liver cancer. ${ }^{17}$ However, whether O-GlcNAcylation is involved in the pathways driving $\mathrm{HB}$ tumor progression and development and whether key regulatory proteins are O-GlcNAcylated and phosphorylated have not been examined.

In this study, we performed large-scale identification of $\mathrm{O}-$ GlcNAcylated and phosphorylated proteins in HB and normal liver tissues and examined the related physiological processes. Our data shed light on the potential key regulatory proteins in HB and helped provide a foundation for the functional study of $\mathrm{O}$ GIcNAcylated or phosphorylated proteins in HB.

\section{RESULTS}

O-GIcNAcylation and OGT levels were upregulated in HB tissues Here, we sought to determine whether O-GlcNAcylation played a critical role in the development of $\mathrm{HB}$. Western blot and immunohistochemical analyses were performed in 4 paired $\mathrm{HB}$ tissues and matched noncancerous normal tissues. The results showed that the levels of both total O-GIcNAcylation and OGlcNAc transferase (OGT) were elevated in all HB tissues compared with those in the matched normal tissues (Fig. 1a, b).

O-GlcNAcylation and phosphorylation in the proteome of $\mathrm{HB}$ and normal tissues

The high levels of O-GICNAc modification in HB tissues suggested that O-GlcNAcylation might be associated with the pathogenesis of HB. Previous studies have shown that O-GlcNAcylation and phosphorylation can target the same Ser/Thr site(s) or different Ser/Thr sites. ${ }^{15}$ Hence, it is essential to identify O-GlcNAcylated and/or phosphorylated sites and proteins and determine their potential regulatory role in HB. Thus, we employed the workflow of PTM Bio (Zhejiang, China) to identify and compare OGlcNAcylated and phosphorylated proteins in the abovementioned 4 pairs of $\mathrm{HB}$ and normal matched tissues (Fig. 1c). Proteins were extracted from tissues, and O-GICNAc-modified or phosphomodified peptides were enriched using O-GIcNAc antibody beads and Immobilized metal affinity chromatography (IMAC). Peptide samples were processed by tandem mass spectrometry (MS/MS), and MaxQuant was used to analyze the MS/MS data. The
Table 1. MS/MS spectrum database search analysis summary

\begin{tabular}{lll}
\hline Modification & O-GlcNAcylation & Phosphorylation \\
\hline Total spectrums & 39,635 & 114,007 \\
Matched spectrum & $2046(5.2 \%)$ & $23,624(20.7 \%)$ \\
Peptides & 1223 & 6254 \\
Modified peptides & 114 & 3494 \\
Identified proteins & 78 & $2088(1894)$ \\
Identified sites & 141 & $4546(3372)$ \\
\hline
\end{tabular}

reproducibility of quantitative proteomic measurements among the four patient samples was precisely evaluated, and the results are shown in Supplementary Table S1 and Supplementary Data S1 and Supplementary Data S2.

The data analysis identified 114 differentially expressed OGlcNAcylated sites mapping to 78 proteins from both HB tissues and normal tissues and 3494 unique phosphorylation sites in 2088 proteins (Table 1). Details of the modified sites and peptides are listed in Supplementary Data S3 and Supplementary Data S4.

Overview of the identified O-GIcNAcylated and phosphorylated peptides and proteins

To more closely examine the characteristics of the modified sites, we performed motif analysis on the flanking sequences of these sites. In O-GlcNAcylated proteins, there was a preference for threonine residues at the -3 position (Fig. 2a). In phosphorylated proteins,...R..SP.P...,.....SP...R.,...R..SP.... and.....SP.R... were characterized as the top four upregulated motifs for phosphorylation sites (Fig. S1a). Details of the motifs in phosphorylated peptides are shown in Supplementary Data S5.

WoLF PSORT was used to predict the subcellular localization of the identified O-GIcNAcylated or phosphorylated proteins. The majority of the detected O-GIcNAcylated or phosphorylated proteins were predicted to accumulate in the nucleus, cytoplasm, extracellular space, mitochondria, and endoplasmic reticulum. A few phospho-modified proteins tended to be localized in the peroxisome, cytoskeleton, and Golgi apparatus (Fig. 2b, Fig. S1b). 
a

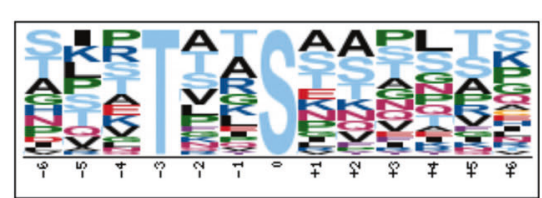

b

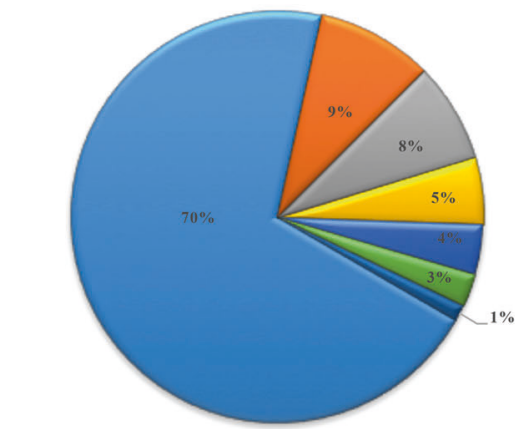

nucleus

田extracellular

cyto nucl

endoplasmic reticulum

d

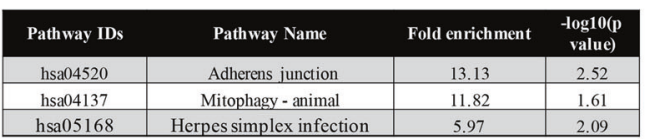

c

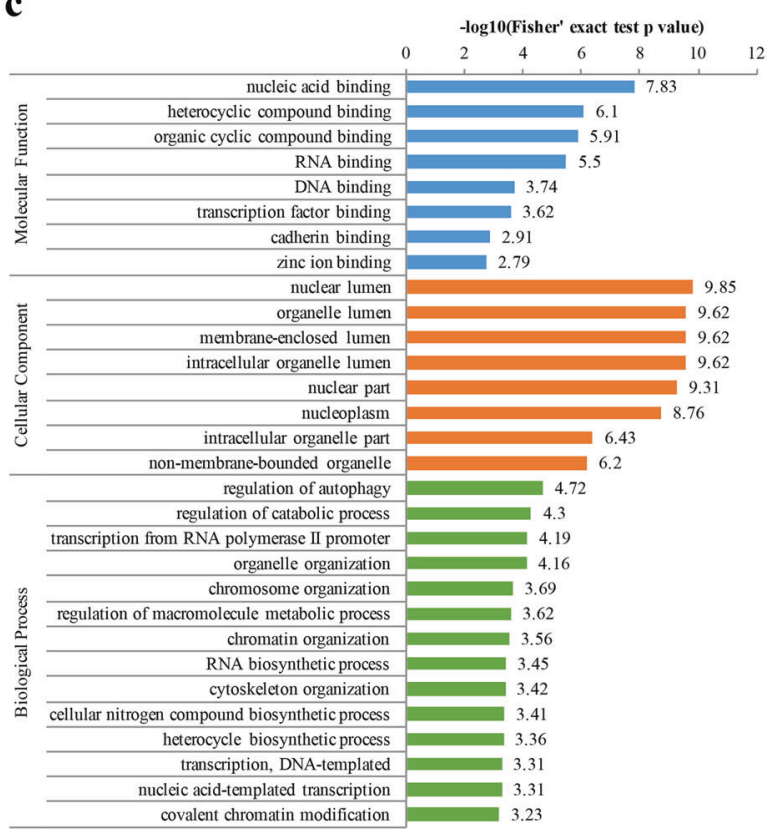

e

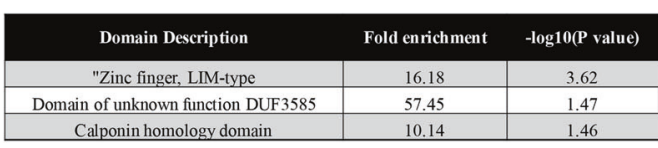

Fig. 2 Summary of identified O-GIcNAcylated proteins and peptides. a Motif analysis of O-GlcNAcylated sites identified in this study. b Subcellular localization of identified O-GlcNAcylated proteins. c Gene Ontology (GO) analysis of O-GlcNAcylated proteins. d KEGG pathway analysis of all O-GlcNAcylated proteins. e Protein domain enrichment analysis of O-GlcNAcylated proteins

Gene Ontology (GO) enrichment analysis was performed to elucidate the cellular components, molecular functions and biological processes of O-GlcNAcylated or phosphorylated proteins. The results indicated that O-GIcNAc-modified and phosphomodified proteins are components of the nuclear and organelle lumen and the cell junctions, respectively. In addition, $\mathrm{O}$ GlcNAcylated proteins are involved in nucleic acid binding and are enriched in the terms regulation of autophagy, catabolic process, and transcription from RNA polymerase II (Fig. 2c). Unlike O-GlcNAcylated proteins, phospho-modified proteins were enriched in RNA binding and associated with mRNA processing and RNA splicing (Fig. S1c).

KEGG pathway annotation revealed that O-GlcNAcylated proteins were related to adherens junction and mitophagy (Fig. 2d), whereas phosphorylated proteins were associated with multiple signaling pathways, including the spliceosome, tight junction and glucagon pathways (Fig. S1d). Protein domain enrichment analysis showed that zinc finger, LIM-type, domain of unknown function DUF3585 and calponin homology domains were enriched in O-GlcNAcylated proteins (Fig. 2e). Phosphorylated proteins were enriched in nucleotide-binding alpha-beta plait domains, RNA recognition motif domains and pleckstrin homology domains (Fig. S1e).

\section{Functions of O-GlcNAcylated proteins}

While numerous studies have elucidated the functions of phosphorylated proteins, little is known about the role of O-GlcNAcmodified proteins. As illustrated in Fig. 3, O-GlcNAcylated proteins identified in this study were associated with multiple biological processes. Many of the identified O-GIcNAcylated proteins, including histone acetyltransferase p300 (EP300) and the nucleosomeremodeling factor subunit BPTF, were related to chromatin remodeling. The majority of these proteins are transcription and translation modifiers that participate in mRNA elongation (AFF4 ${ }^{20}$ and $\left.\mathrm{CDK}_{12} 2^{21}\right)$, degradation (KHSRP ${ }^{22}$ ), splicing (SRRM1 ${ }^{23}$ ) and deadenylation $\left(\mathrm{RC} \mathrm{H}_{2}{ }^{24}\right)$. These proteins are also involved in organelle organization-for example, the critical components of transitional ER organization, SEC $16 \mathrm{~A}^{25}$ and KRT18, which play a key role in the reorganization of intermediate filaments. ${ }^{26}$ In addition, several O-GlcNAcylated proteins were linked to the regulation of autophagy and carcinogenesis. A few O-GlcNAc-modified proteins were associated with metabolism, transportation and the nervous system.

Crosstalk between O-GlcNAcylation and phosphorylation The distribution of the number of modified sites identified per protein is shown in Fig. 4a, b. The majority of the O-GlcNAcylated or phosphorylated proteins had only 1 modified site. In addition, $\log 2$ transformation was performed to quantify the modification sites on the modified proteins, and most of the ratios $(T / N)$ were between 0 and 1 . In addition, our data revealed that $52 \%$ of the identified O-GIcNAcylated proteins were also modified by phosphorylation and that these proteins were related to chromatin regulation, transcription, translation, transportation, and organelle organization. To more closely examine the interaction networks among these proteins, protein complex enrichment analysis was performed. These results showed that some of the proteins modified by both O-GIcNAcylation and phosphorylation could interact with each other (Fig. 4c) and thus might play a synergistic role in cellular signal transduction.

\section{Verification of the MS data}

Among the proteins modified by both O-GlcNAcylation and phosphorylation, NPM1, HSPE1, and HSPB1 attracted our attention, as these proteins are involved in tumorigenesis. ${ }^{27-29} \mathrm{We}$ further verified the accuracy of the MS data by performing 
Chromatin Regulation

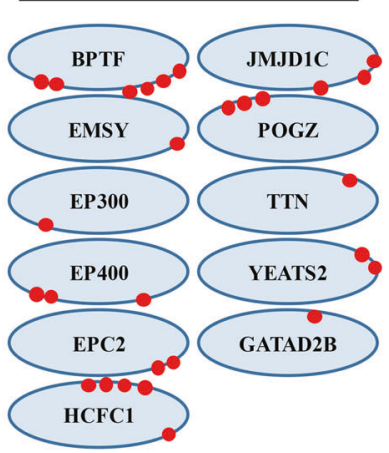

Carcinogenesis

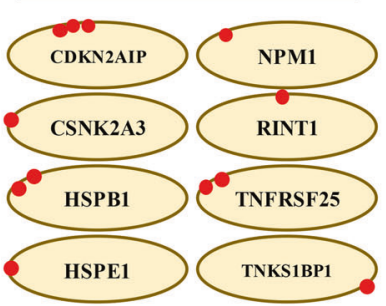

Transcription \& Translation
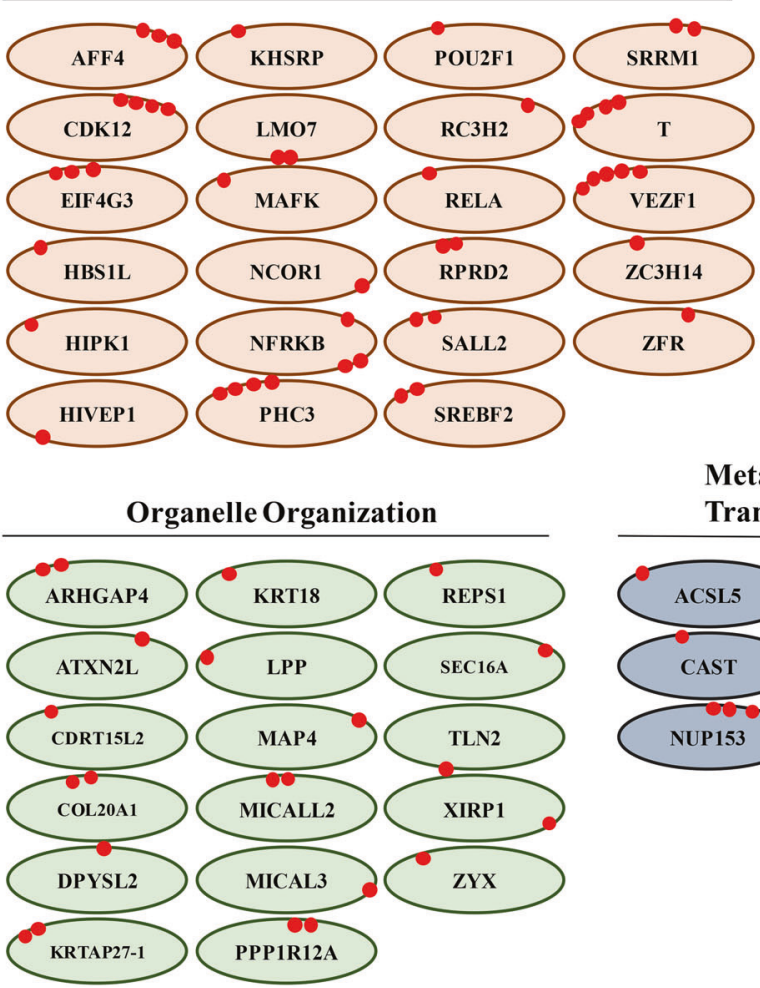

Metabolism \&

Transportation Nervous system

Autophagy
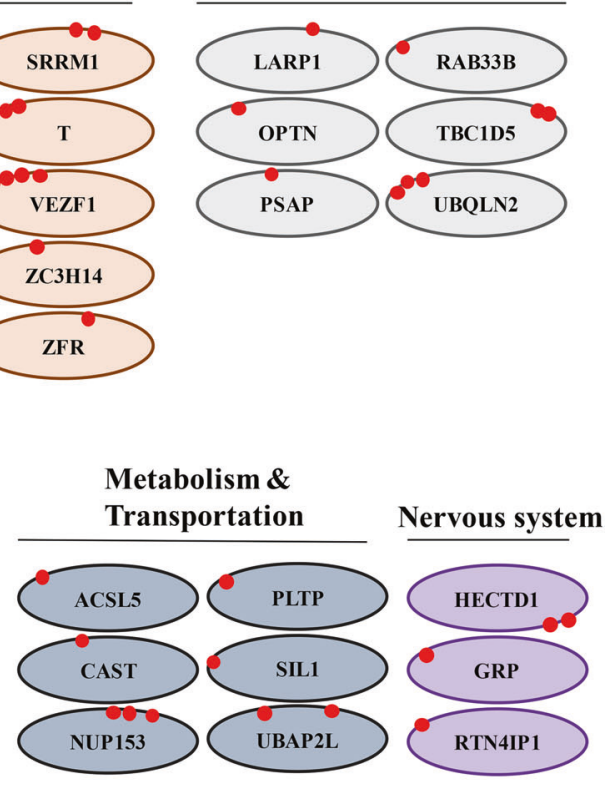

Fig. 3 Functions of O-GlcNAcylated proteins. Proteins with O-GlcNAcylation modification were classified into several groups according to their known functions. Each red dot represents an O-GlcNAc-modified site

immunoprecipitation and western blot analysis in HB tissues. These results showed that NPM1, HSPE1, and HSPB1 were modified by both O-GIcNAcylation and phosphorylation, and the levels of NPM1, HSPE1, and HSPB1 O-GlcNAcylation and phosphorylation were significantly higher in $\mathrm{HB}$ tissues than in normal tissues (Fig. 5a, b).

HSPB1 is O-GlcNAc-modified and associated with the survival and chemotherapy resistance of $\mathrm{HB}$ cell lines

Previous studies demonstrated that heat shock protein beta- 1 (HSPB1) is abnormally expressed in various human cancers, including glioma, ${ }^{30}$ hepatocellular carcinoma, ${ }^{31}$ non-small-cell lung cancer ${ }^{32}$, and breast cancer. ${ }^{33}$ However, the expression and function of HSPB1 in HB are not known. Western blotting and immunohistochemistry revealed higher expression of HSPB1 in HB tissues than in the matched normal tissues (Fig. 6a, b). Consistent with the results in $\mathrm{HB}$ tissues, O-GlcNAcylation and phosphorylation modification of HSPB1 were observed in the HB cell lines HepG2 and Huh6 (Fig. 6c). It has been reported that phosphorylation of the serine residue at position 82 contributes to the diverse functional roles of HSPB1. ${ }^{34-36}$ We next examined whether there was potential competition between O-GlcNAcylation and phosphorylation of Ser82 in HSPB1. HB cells transfected with MycHSPB1-WT were treated with PUGNAC $(25 \mathrm{mM})$ and GLCNAC $(4 \mathrm{mM})$ or with P79350 $(50 \mathrm{nM})$ to increase the levels of OGIcNAcylation or phosphorylation of HSPB1 Ser82, respectively. Interestingly, the data demonstrated that the levels of HSPB1 OGlcNAcylation and phosphorylation in $\mathrm{HB}$ cells were dynamically changed. As shown in Fig. 6d, we observed increased levels of OGlcNAc-modified HSPB1 after stimulation toward O-GlcNAcylation, with a parallel decrease in HSPB1 phosphorylation (Ser82) in both HepG2 and Huh6 cells.

The MS data suggested that 180S and 187T in HSPB1 were modified by O-GlcNAcylation. To confirm whether these PTMs occurred at $180 \mathrm{~S}$ and/or $187 \mathrm{~T}$ in HSPB1, we generated a panel of plasmids expressing point mutations of HSPB1 (Myc-HSPB1-T180A, Myc-HSPB1-S187G, and Myc-HSPB1-T180A/S187G). We transfected cells with the mutant plasmids and exposed the cells to P79350 $(50 \mathrm{nM})$ or to PUGNAC $(25 \mathrm{mM})$ and GLCNAC $(4 \mathrm{mM})$ for $0.5 \mathrm{~h}$ or $48 \mathrm{~h}$, respectively. Myc immunoprecipitation was performed, followed by western blotting. While a strong increase in O-GlcNAcylation of wildtype HSPB1 was observed, the T180A, S187G, and T180A/S187G mutants showed relatively reduced levels of O-GlcNAcylation, suggesting that HSPB1 might be O-GlcNAcylated at S180 and T187. Hence, we sought to determine whether the crosstalk between the O-GlcNAcylation and phosphorylation of Ser82 was mediated by O-GlcNAcylation of Ser180 and/or Tyr187. However, mutation of Ser180 and/or Tyr187 had little effect on the phosphorylation of Ser82 in HB cells with or without stimulation toward O-GlcNAcylation (Fig. 6e), indicating that other sites in HSPB1 are O-GlcNAc-modified. Notably, the proliferation experiment showed that overexpressing HSPB1 or increasing the O-GlcNAcylation of HSPB1 alone increased cell proliferation, whereas advanced O-GlcNAcylation of HSPB1 displayed a superior ability for cell multiplication in tumor progression (Fig. 6f, g). In addition, O-GlcNAcylation of HSPB1 stimulated the tolerance of HB cells to cisplatin in vitro. These results further suggested that HSPB1 might serve as a therapeutic target in HB (Fig. 6h).

\section{DISCUSSION}

Multiple proteomic studies have shown that O-GlcNAcylation and phosphorylation modifications serve as key regulators of various physiological functions. ${ }^{6-8,10-13}$ Previous research has identified and characterized a large set of O-GlcNAcylated or phosphorylated proteins in murine synapses and Arabidopsis. ${ }^{18,19}$ In addition, the role of O-GIcNAcylation and phosphorylation has been examined for specific proteins. However, a comprehensive investigation and 
a The numeber of phosphorylated sites identifications per protein

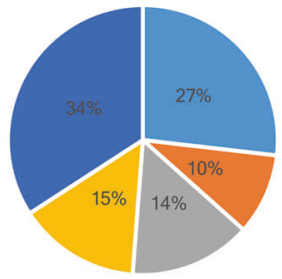

- site $>=5 \quad$ a site $=4 \quad$ " site $=3 \quad$ a site $=2 \quad$ a site $=1$

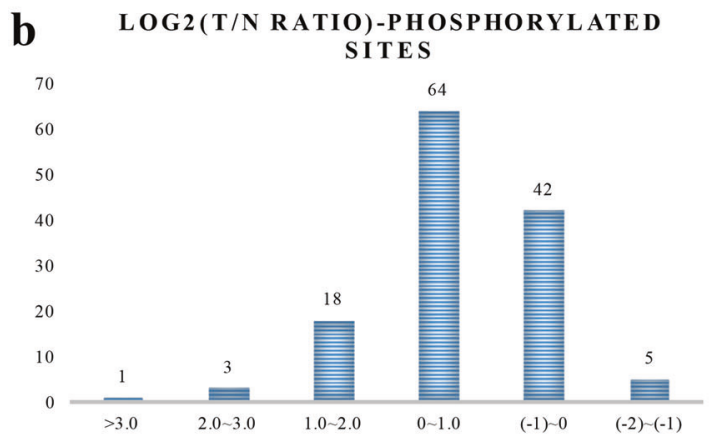

The numeber of O-glcnac sites identifications per protein

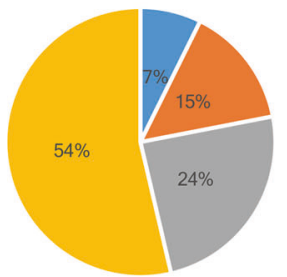

site $>=4$ " site $=3 \quad$ " site $=2 \quad$ "site $=1$

LOG 2(T/N RATIO)-O-GLCNACYLATED

S I T ES

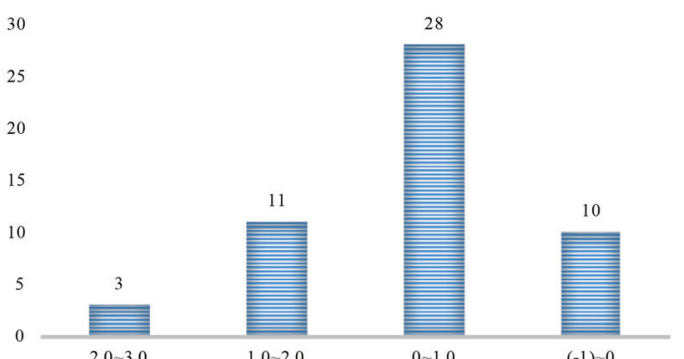

C

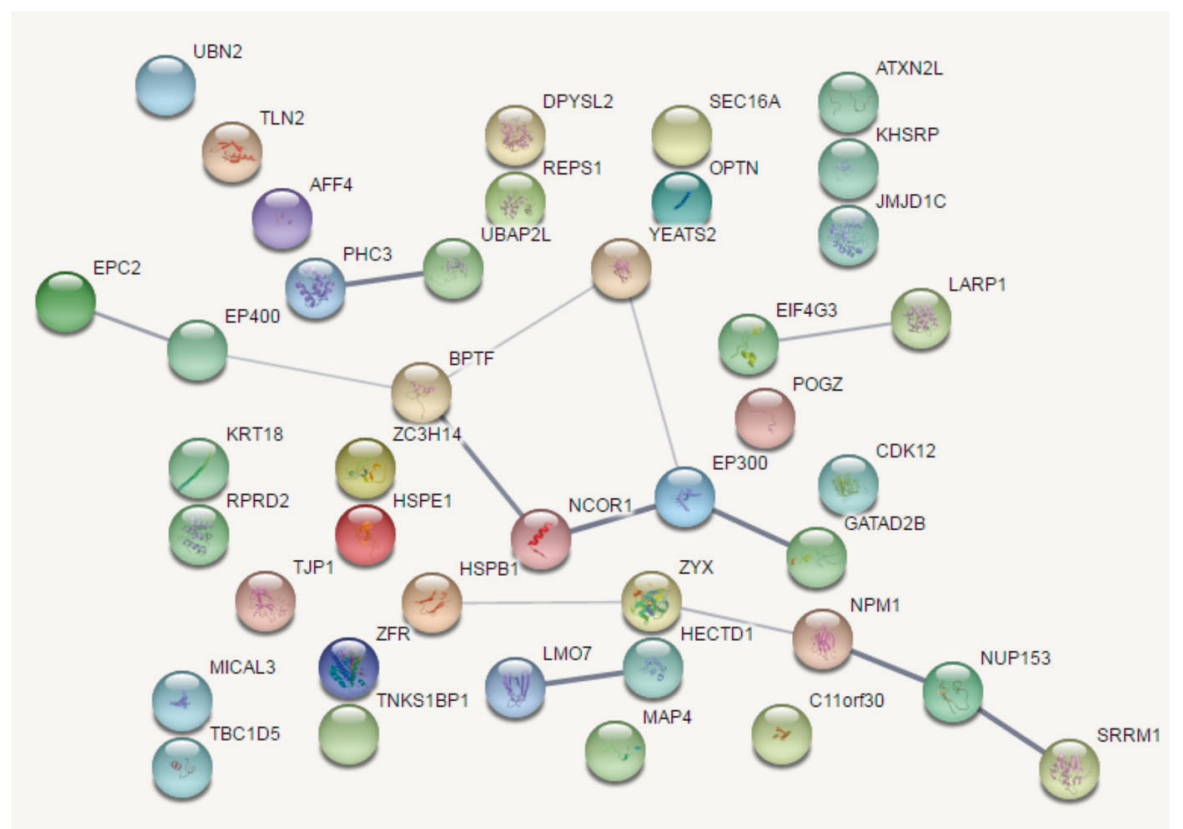

Fig. 4 Overview of proteins and peptides modified by both O-GlcNAcylation and phosphorylation. a Numerical distribution of modified sites. b Quantitative distribution of modified sites. c Protein-protein interaction network of proteins modified by both O-GlcNAcylation and phosphorylation

survey of both O-GlcNAcylation and phosphorylation in human tumors has not been conducted, especially in $\mathrm{HB}$, a rare disease with a largely unknown pathogenesis. ${ }^{1-3}$ Our study is the first to examine the O-GlcNAcylated and phosphorylated proteins in HB. GO and KEGG analyses uncovered multiple functions for these proteins in various physiological and biochemical processes. These data provide a broad basis and foundation for further research on the functional consequence of the O-GlcNAcylation or phosphorylation of specific proteins in $\mathrm{HB}$ and other kinds of human tumors.
Interestingly, our results demonstrated that the sequence preference of the identified O-GICNAc-modified or phosphomodified sites was dissimilar to that found in other diseases and plants, indicating that the enzymatic characteristics of OGTs and protein kinases are distinct in different microenvironments. The majority of the modified proteins in our project were predicted to localize in the nucleus and cytoplasm, and functional analysis suggested that these proteins were involved in mRNA transcription, translation and chromatin remodeling. These data demonstrated 

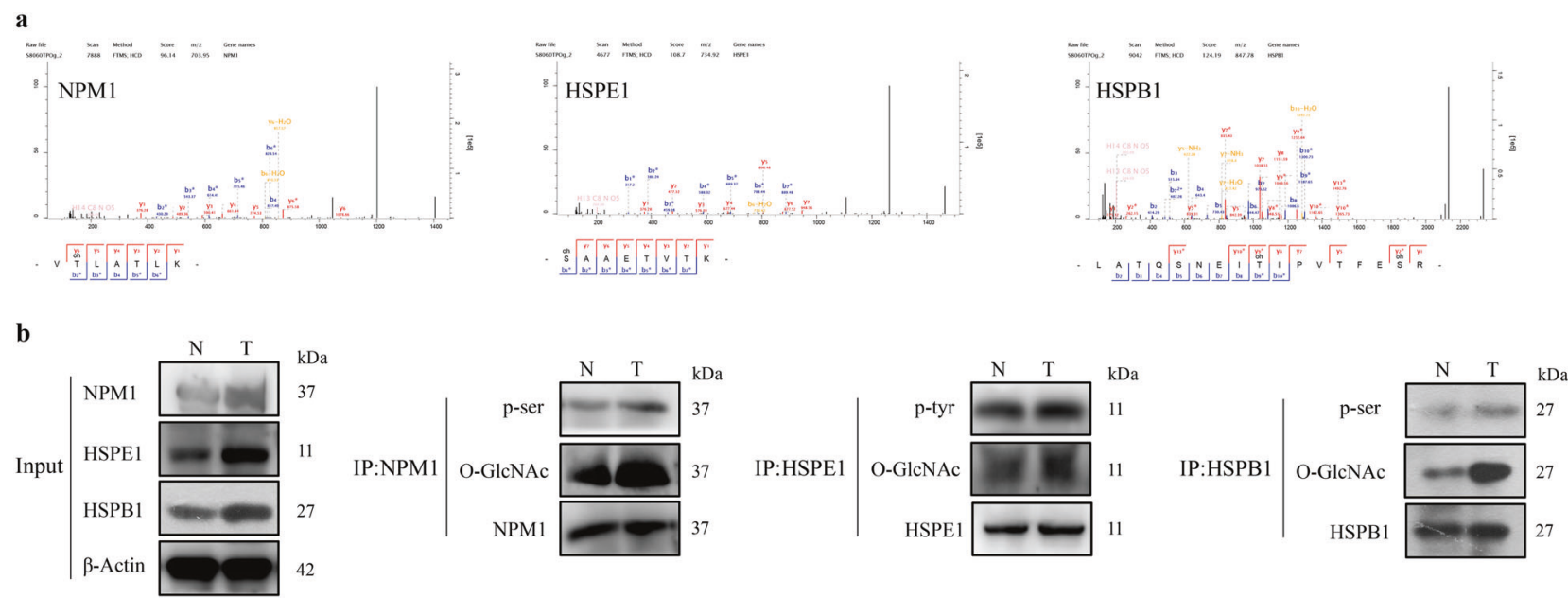

Fig. 5 NPM1, HSPE1, and HSPB1 were both O-GICNAc-modified and phospho-modified. a ETD mass spectra of peptides derived from NPM1, HSPE1, and HSPB1. b The O-GlcNAcylation and phosphorylation levels of endogenous NPM1, HSPE1, and HSPB1 were measured in HB tissues and normal tissues by immunoprecipitation and western blot analysis. All experiments were repeated three times

that the locations and functions of the identified O-GlcNAcylated or phosphorylated proteins were similar to those of proteins in plants, ${ }^{19}$ revealing the evolutionary conservation of these proteins.

Our data revealed 114 differentially expressed O-GlcNAcmodified sites in 78 proteins and 3494 phosphorylation sites in 2088 proteins. These numbers are slightly lower than those in the dataset of modified proteins in other tissues. ${ }^{18,19}$ However, the results of GO and KEGG analyses provided adequate evidence that O-GlcNAcylated or phosphorylated proteins with important biological roles were identified in our project. In addition, we verified the data for a subset of the identified proteins in $\mathrm{HB}$ tissues and cell lines. Mutagenesis studies confirmed that the $\mathrm{O}-$ GlcNAc-modified sites in HSPB1 were consistent with the sites identified via proteomic analysis and that there was crosstalk between the O-GlcNAcylation and phosphorylation of HSPB1. These findings provided evidence for the accuracy of our data.

Several considerations should be noted regarding our study. Notably, our initial analysis failed to identify $\beta$-catenin, which has been shown to be O-GlcNAc-modified or phosphorylated in various kinds of cancers. ${ }^{37-40}$ Further, the number of samples in the present study is small. Therefore, a larger sample of HB tissues and matched normal tissues is required to improve the precision and accuracy of these results. However, our proteomics data provide valuable preliminary information for future functional studies.

Recent reports have shown that autophagy plays a critical role in tumor development and progression by stimulating progression through supplying sufficient amounts of essential nutrients or eliminating damaged or mutated macromolecules in cancer cells. ${ }^{41,42}$ Notably, several O-GlcNAc-modified autophagy proteins were identified in our dataset, including optineurin (OPTN), which is an autophagy modifier in the MAP1 LC3 family. ${ }^{43}$ In addition, our results showed the O-GlcNAcylation of several tumorassociated proteins, such as CDKN2AIP ${ }^{44}$ (a key regulator of DNA damage and repair), CSNK2 ${ }^{45}$ (a tumor growth-promoting factor) and RINT1 ${ }^{46}$ (which functions as a modifier in cell cycle checkpoint control). Our results suggested that these proteins might be involved in the development and progression of $\mathrm{HB}$, and future studies should examine their function, because they may serve as molecular targets in $\mathrm{HB}$.

Given that O-GlcNAcylation and phosphorylation may occur on nearby or on the same Ser/Thr residues, we analyzed the protein interaction network of the O-GlcNAcylated and phosphorylated proteins via the STRING database. We found that proteins that were both O-GlcNAcylated and phosphorylated were involved in critical physiological processes, such as chromatin regulation, transcription and translation. In addition, several of the proteins that were both O-GlcNAcylated and phosphorylated were predicted to interact with each other, suggesting the important role of O-GlcNAcylation and phosphorylation modifications in the regulation of $\mathrm{HB}$.

Taken together, our data provide comprehensive information on the O-GIcNAc-modified and/or phospho-modified peptides and proteins in HB. Notably, the locations and functions of these proteins were evolutionarily conserved. Functional prediction analysis indicated that these modified proteins might serve as critical regulators of diverse cellular and molecular processes. These findings will help further our understanding of the biological function of protein OGlcNAcylation and phosphorylation in HB. Furthermore, our data lay a foundation for research on the crosstalk between protein OGlcNAcylation and phosphorylation in other diseases.

\section{MATERIALS AND METHODS}

Drugs and reagents

Doxorubicin and cisplatin were acquired from Selleck Chemical (TX, USA), PUGNAc and GICNAc were purchased from SigmaAldrich (MO, USA), and P79350 was obtained from Invitrogen (CA, USA). All other reagents and chemicals, unless otherwise stated, were obtained from Beyotime (Jiangsu, China).

Specimens and immunohistochemical staining

Four pairs of cancerous tissues and matched noncancerous normal liver tissues were collected from HB patients who underwent surgery at the Department of Hepatobiliary Surgery of the Shanghai Children's Medical Center. Patients did not receive chemotherapy or radiotherapy before admission. This study was approved by the Ethics Committee of the Shanghai Children's Medical Center, and written informed consent was obtained from each patient. The detailed information of these patients is listed in Supplementary Table S2.

Immunohistochemical staining was performed as previously described. ${ }^{47}$ The primary antibodies used were anti-OGT (D1D8Q) rabbit mAb (\#24083, Cell Signaling Technology, MA, USA), anti-OGlcNAc mouse mAb (PTM-952, PTM Bio, Zhejiang, China), and antiHSPB1 (D6W5V) rabbit mAb (\#95357, Cell Signaling Technology, MA, USA).

\section{Cell culture}

The human HepG2 and Huh6 HB cell lines were purchased from the Cell Bank of the Chinese Academy of Sciences (Shanghai, China). Both cell lines have been authenticated by STR genotyping. HepG2 


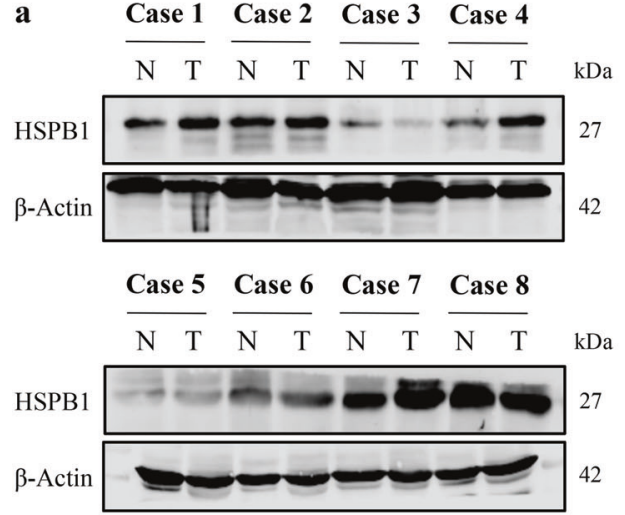

b

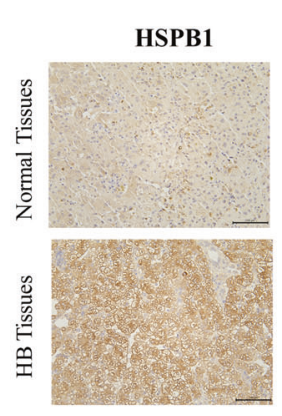

c

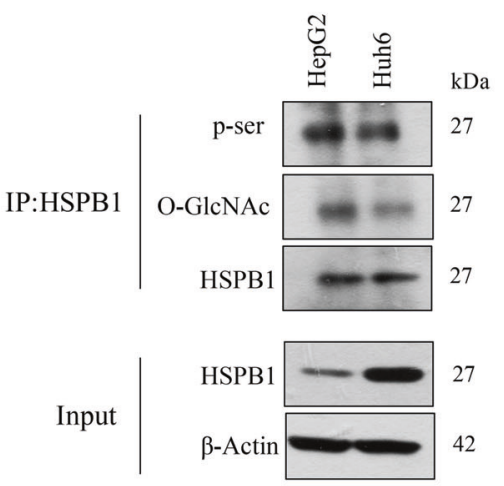

d

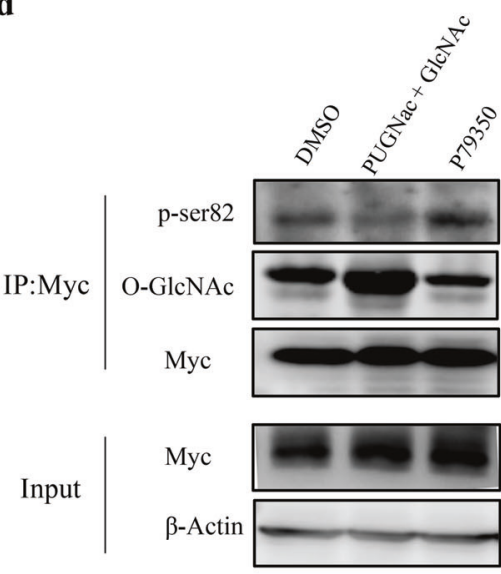

HepG2-WT

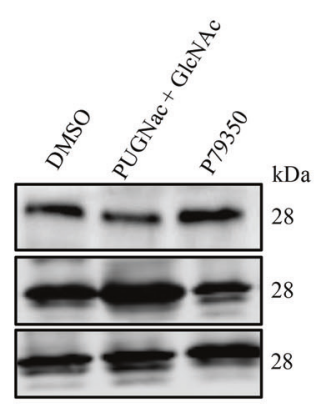

e

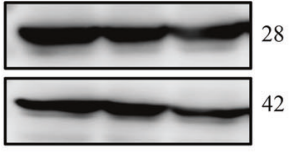

Huh6-WT

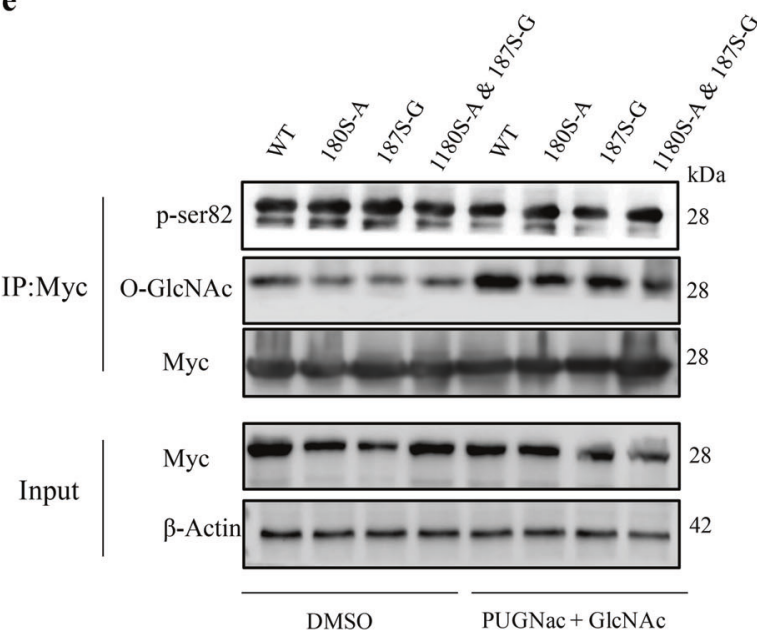

HepG2

f

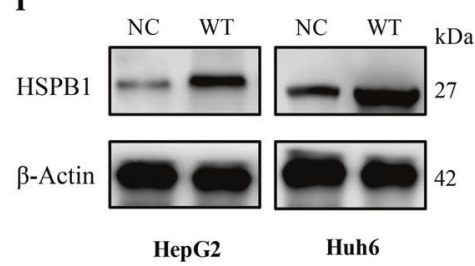

g

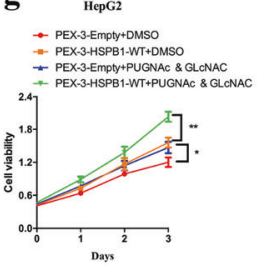

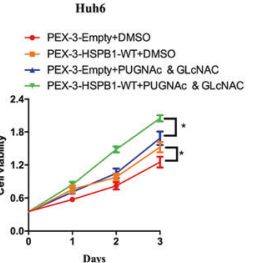

h

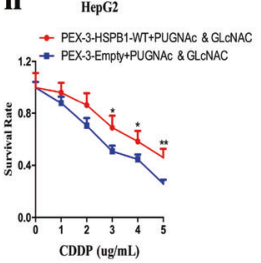

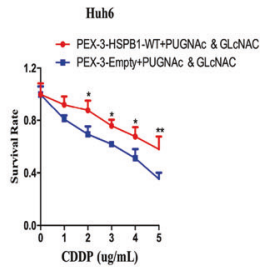

Fig. 6 HSPB1 is O-GlcNAc-modified and involved in the chemotherapy resistance of HB cell lines. a, b Western blotting and immunohistochemistry were performed to determine the expression pattern of HSPB1 in HB tissues and normal tissues. c O-GlcNAcylation and phosphorylation modifications of HSPB1 were detected in HepG2 and Huh6 cells. d Cells were treated with PUGNAc (25 mM) and GLcNAC $(4 \mathrm{mM})$ for $48 \mathrm{~h}$ or with P79350 (50 nM) for $0.5 \mathrm{~h}$, and cell lysates were immunoprecipitated with an anti-Myc antibody, followed by western blotting with either an anti-MYC antibody, anti-O-GlcNAc antibody, or anti-phospho-HSPB1 (Ser82) antibody. e Cells transfected with MycHSPB1-WT or Myc-HSPB1-mutant plasmids were exposed to P79350 $(50 \mathrm{nM})$ or to PUGNAc $(25 \mathrm{mM})$ and GLCNAC (4 mM) for $0.5 \mathrm{~h}$ or $48 \mathrm{~h}$, respectively. Cell lysates were immunoprecipitated with an anti-Myc antibody, followed by western blot analysis. $\mathbf{f}$ Cells were transfected with Myc-HSPB1-WT plasmid, and western blotting was conducted to evaluate the transfection efficiency $48 \mathrm{~h}$ later. $\mathbf{g}$ CCK-8 proliferation assays were applied to assess the effect of HSPB1 O-GlcNAcylation on the survival of HB cells. $\mathbf{h}$ CCK- 8 proliferation assays were performed to evaluate the effect of HSPB1 O-GlcNAcylation on cisplatin tolerance. The error bars indicate the SD, ${ }^{*} p<0.05 ;{ }^{* *} p<0.01$. All experiments were repeated three times

and Huh6 cells were cultured in minimum essential medium (MEM, HyClone, UT, USA) and Dulbecco's modified Eagle's medium (DMEM, HyClone, UT, USA), respectively, supplemented with $10 \%$ fetal bovine serum (Gibco, CA, USA), $100 \mathrm{U} / \mathrm{ml}$ streptomycin (Invitrogen, CA, USA) and $100 \mathrm{U} / \mathrm{ml}$ penicillin (Invitrogen, CA, USA) at $37^{\circ} \mathrm{C}$ in a humidified atmosphere with $5 \% \mathrm{CO}_{2}$.

Western blot analysis

Tissues and cells were lysed in western lysis buffer (Beyotime, Jiangsu, China) for $30 \mathrm{~min}$ on ice, followed by centrifugation at
$12,000 \times g$ for $15 \mathrm{~min}$. Protein samples $(30 \mu \mathrm{g})$ were separated on SDS-PAGE gels and transferred to PVDF membranes (GE Healthcare, Buckinghamshire, UK). Membranes were blocked with $5 \%$ BSA at room temperature for $1 \mathrm{~h}$, followed by overnight incubation with primary antibodies at $4{ }^{\circ} \mathrm{C}$. Membranes were washed with PBST three times, followed by incubation with the appropriate HRP-conjugated secondary antibodies (Santa Cruz, CA, USA) at room temperature for $1 \mathrm{~h}$. Bands were visualized using a SuperSignal West Femto kit (Pierce, IL, USA). GAPDH was used as the loading control. The primary antibodies used were anti-OGT 
(D1D8Q) rabbit mAb (\#24083, Cell Signaling Technology, MA, USA), anti-O-GlcNAc mouse mAb (PTM-952, PTM Bio, Zhejiang, China), anti-HSPB1 (D6W5V) rabbit mAb (\#95357, Cell Signaling Technology, MA, USA), anti-Phospho-HSPB1 (Ser82) (D1H2F6) rabbit mAb (\#9709, Cell Signaling Technology, MA, USA), antiNPM1 rabbit pAb (10306-1-AP, Proteintech, IL, USA), anti-HSPE1 rabbit mAb (ab108600, Abcam, MA, USA), anti-phosphoserine/ threonine/tyrosine mouse mAb (ab15556, Abcam, MA, USA), antiMyc-Tag (9B11) mouse mAb (\#2276S, Cell Signaling Technology, MA, USA) and anti-phospho-(Ser) Arg-X-Tyr/Phe-X-pSer motif rabbit Ab (\#2981S, Cell Signaling Technology, MA, USA).

\section{Immunoprecipitation}

Tissues and cells were lysed in IP lysis buffer (Beyotime, Jiangsu, China), and $500 \mu \mathrm{g}$ samples of total protein were incubated with specific antibodies and protein A/G PLUS agarose beads (Santa Cruz, CA, USA)) overnight at $4^{\circ} \mathrm{C}$. The beads were washed with IP lysis buffer, and western blotting was then performed. The antibodies used were anti-Myc (9B11) mouse mAb (\#2276S, Cell Signaling Technology, MA, USA), anti-HSPB1 (D6W5V) rabbit mAb (\#95357, Cell Signaling Technology, MA, USA), anti-NPM1 rabbit pAb (10306-1-AP, Proteintech, IL, USA) and anti-HSPE1 rabbit mAb (ab108600, Abcam, MA, USA).

\section{Sample preparation for LC-MS/MS}

Total protein was extracted from tissues using lysis buffer (PTM Bio, Zhejiang, China). Samples were sonicated at least three times on ice using an ultrasonic processor (Scientz, Zhejiang, China). To obtain peptides, samples were reduced with DIT and alkylated with iodoacetamide in the dark at room temperature, digested with trypsin, desalted using a Strata X C18 SPE column (Phenomenex, CA, USA) and vacuum-dried. Peptides were reconstituted and processed with a TMT kit/iTRAQ kit according to the manufacturer's instructions.

HPLC fractionation and enrichment of O-GlcNAcylated peptides and phosphorylated peptides

Peptide fractions were acquired on a Thermo Betasil C18 column ( $5 \mu \mathrm{m}$ particles, $10 \mathrm{~mm} \mathrm{ID,} 250 \mathrm{~mm}$ length) through high $\mathrm{pH}$ reversed-phase HPLC. For enrichment of O-GICNAc-modified peptides, tryptic peptides were incubated with prewashed OGlcNAc antibody beads (PTM-954, PTM Bio, Zhejiang, China) and incubated in NETN buffer ( $100 \mathrm{mM} \mathrm{NaCl}, 1 \mathrm{mM}$ EDTA, $50 \mathrm{mM}$ Tris$\mathrm{HCl}, 0.5 \% \mathrm{NP}-40(\mathrm{pH} 8.0))$ at $4{ }^{\circ} \mathrm{C}$ with gentle shaking overnight. Immunocomplexes were washed with NETN buffer four times and were then washed with double distilled water. We used $0.1 \%$ trifluoroacetic acid to elute the bound fractions from the beads. The collected peptides were vacuum dried, followed by desalting with C18 ZipTips (Millipore, MA, USA) according to the manufacturer's instructions. For enrichment of phospho-modified peptides, tryptic peptide mixtures were mixed with IMAC microspheres in loading buffer (50\% acetonitrile/6\% trifluoroacetic acid) with gentle vibration. After centrifugation, the supernatant was removed, and IMAC microspheres with bound phosphopeptides were acquired. These IMAC microspheres were washed with $50 \%$ acetonitrile/6\% trifluoroacetic acid and 30\% acetonitrile/0.1\% trifluoroacetic acid continuously to remove nonspecifically adsorbed peptides. The phosphopeptides were then eluted from the IMAC microspheres in elution buffer with vibration. Finally, the peptides were lyophilized for LC-MS/MS analysis.

\section{LC-MS/MS analysis}

Formic acid $(0.1 \%$, solvent A) containing the O-GlcNAc-modified peptides or phospho-modified peptides was loaded onto a reversed-phase analytical column $(15 \mathrm{~cm}$ length, $75 \mu \mathrm{m}$ ID). A concentration gradient was used with an increase from $6 \%$ to $23 \%$ $0.1 \%$ formic acid in $98 \%$ acetonitrile (solvent B) for $26 \mathrm{~min}$; an increased from $23 \%$ to $35 \%$ for $8 \mathrm{~min}$; an increase to $80 \%$ for
$3 \mathrm{~min}$; and maintenance at $80 \%$ for the last $3 \mathrm{~min}$. The flow rate was constant at $400 \mathrm{~nL} / \mathrm{min}$ on an EASY-nLC 1000 UPLC system.

Peptides were first exposed to an NSI source and were then analyzed by tandem mass spectrometry (MS/MS) in a $Q$ Exactive ${ }^{T M}$ Plus (Thermo, MA, USA) coupled to a UPLC system. The specific parameters were as follows: a $2.0 \mathrm{kV}$ electrospray voltage was used; the m/z scan was set from 350 to 1800; and the resolution of the Orbitrap was set as 70,000 to detect intact peptides. Next, the NCE (set to 28) and Orbitrap (resolution $=17500$ ) were used to select peptides for MS/MS and detect the fragments. The datadependent procedure alternated between one MS scan followed by $20 \mathrm{MS} / \mathrm{MS}$ scans with the automatic gain control set at $5 \mathrm{E} 4$ and a fixed first mass of $100 \mathrm{~m} / \mathrm{z}$.

\section{Database search}

We used the MaxQuant search engine (v.1.5.2.8) to analyze the MS/ MS data. The tandem mass spectra were searched against the SwissProt Human database concatenated with a reverse decoy database. The data were also searched with a common contamination database to eliminate the impact of contaminated proteins. Trypsin/P was specified as the cleavage enzyme, allowing up to two missed cleavages. The mass tolerance for precursor ions in the first search and the main search was set to $20 \mathrm{ppm}$ and $5 \mathrm{ppm}$, respectively. The mass tolerance for fragment ions was set to $0.02 \mathrm{Da}$. Cysteine carbamidomethylation was specified as the fixed modification. Oxidation of methionine, acetylation of $\mathrm{N}$-terminal proteins and O-GIcNAcylation of serine and threonine were specified as variable modifications. The false discovery rate for protein and peptide-to-spectrum match identification was adjusted to $1 \%$.

\section{Plasmids and transfection}

Myc-tagged wild-type HSPB1 and mutants, including Myc-HSPB1T180A, Myc-HSPB1-S187G, and Myc-HSPB1-T180A/S187G, were chemically synthesized by GenePharma (Shanghai, China) and subcloned into the pEX-3 expression plasmid (GenePharma, Shanghai, China). All vectors were tested and confirmed by DNA sequencing. Cells were transfected with overexpression plasmids using ExFect Transfection Reagent (Vazyme, Jiangsu, China) in accordance with the manufacturer's instructions. Western blotting was conducted to evaluate the transfection efficiency $48 \mathrm{~h}$ later.

Cell counting Kit-8 (CCK-8) assay

Cells were seeded into 96 -well plates at a density of 5000 cells per well. After treatment, $10 \mu \mathrm{l}$ of CCK-8 reagent (Beyotime, Jiangsu, China) was added, and cells were incubated at $37^{\circ} \mathrm{C}$ for $4 \mathrm{~h}$. The absorbance of each well was detected at a wavelength of $450 \mathrm{~nm}$ by a multimode plate reader.

Statistical analysis

All data were processed by GraphPad Prism 6 software (CA, USA) and are presented as the means \pm SDs. Student's $t$ test was performed to analyze the differences between groups. In all experiments, $p<0.05$ was considered statistically significant.

\section{ACKNOWLEDGEMENTS}

This study was financially supported by the National Natural Science Foundation of China (81572330, 81802103, 81772941 and 81871727), a Municipal Human Resources Development Program for Outstanding Leaders in Medical Disciplines in Shanghai (2017BR036), the Program of Shanghai Academic/Technology Research Leaders (18XD1402600), Shanghai Municipal Education Commission-Gaofeng Clinical Medicine Grant Support (20171926) and the Science and Technology Development Fund of the Pudong New Area of Shanghai (PKJ2017-Y03).

\section{AUTHOR CONTRIBUTIONS}

Conceptualization: Q.P., F.S., J.M. and M.X.; methodology: M.Y., H.S. and Z.B.; investigation: H.S. and S.C.; validation: J.Z., J.M., J.W. and N.H.; writing-review and editing: H.S. and Z.B. 


\section{ADDITIONAL INFORMATION}

The online version of this article (https://doi.org/10.1038/s41392-019-0067-4) contains supplementary material, which is available to authorized users.

Competing interests: The authors declare no competing interests.

\section{REFERENCES}

1. Allan, B. J. et al. Predictors of survival and incidence of hepatoblastoma in the paediatric population. Hpb 15, 741-746 (2013).

2. Green, A. L., Furutani, E., Ribeiro, K. D. \& Galindo, C. R. Death within 1 month of diagnosis in childhood cancer: an analysis of risk factors and scope of the problem. J. Clin. Oncol. 35, 1320-1327 (2017).

3. Czauderna, P. et al. Hepatoblastoma state of the art: pathology, genetics, risk stratification, and chemotherapy. Curr. Opin. Pediatr. 26, 19-28 (2014).

4. Wells, L., Whelan, S. A. \& Hart, G. W. O-GlcNAc: a regulatory post-translational modification. Biochem. Biophys. Res. Commun. 302, 435-441 (2003).

5. Zachara, N. E. \& Hart, G. W. O-GICNAc modification: a nutritional sensor that modulates proteasome function. Trends Cell Biol. 14, 218-221 (2004).

6. Zhao, L., Shah, J. A., Cai, Y. \& Jin, J. O-GlcNAc code' mediated biological functions of downstream proteins. Molecules 23, e1967 (2018).

7. Slawson, C., Copeland, R. J. \& Hart, G. W. O-GIcNAc signaling: a metabolic link between diabetes and cancer? Trends Biochem. Sci. 35, 547-555 (2010).

8. Singh, J. P., Zhang, K., Wu, J. \& Yang, X. O-GlcNAc signaling in cancer metabolism and epigenetics. Cancer Lett. 356, 244-250 (2015).

9. Duan, F. et al. O-GlcNAcylation of RACK1 promotes hepatocellular carcinogenesis. J. Hepatol. 68, 1191-1202 (2018).

10. Chen, Q. et al. Tyrosine phosphorylation of LRP6 by Src and Fer inhibits Wnt/ B-catenin signalling. EMBO Rep. 15, 1254-1267 (2014).

11. Imami, K. et al. Phosphorylation of the ribosomal protein RPL12/uL11 affects translation during mitosis. Mol. Cell. 72, 84-98 (2018).

12. Shyhchang, N., Daley, G. Q. \& Cantley, L. C. Stem cell metabolism in tissue development and aging. Development. 140, 2535-2547 (2013).

13. Nikolaienko, O., Eriksen, M. S., Patil, S., Bito, H., \& Bramham, C. R. Stimulus-evoked ERK-dependent phosphorylation of activity-regulated cytoskeleton-associated protein (Arc) regulates its neuronal subcellular localization. Neuroscience. https:// doi.org/10.1016/j.neuroscience.2017.07.026 (2007).

14. Esmaeili, M. A., Farimani, M. M. \& Kiaei, M. Anticancer effect of calycopterin via $\mathrm{PI} 3 \mathrm{~K} / \mathrm{Akt}$ and MAPK signaling pathways, ROS-mediated pathway and mitochondrial dysfunction in hepatoblastoma cancer (HepG2) cells. Mol. Cell Biochem. 397, 17-31 (2014).

15. Butkinaree, C., Park, K. \& Hart, G. W. O-linked beta-N-acetylglucosamine (OGICNAc): extensive crosstalk with phosphorylation to regulate signaling and transcription in response to nutrients and stress. Biochim. Biophys. Acta 1800 96-106 (2010).

16. Qiao, Y. et al. High glucose stimulates tumorigenesis in hepatocellular carcinoma cells through AGER-dependent O-GIcNAcylation of c-Jun. Diabetes. 65, 619-632 (2016).

17. Zhang, X. et al. The essential role of YAP O-GIcNAcylation in high-glucose-stimulated liver tumorigenesis. Nat. Commun. https://doi.org/10.1038/ncomms15280 (2017).

18. Trinidad, J. C. et al. Global identification and characterization of both OGIcNAcylation and phosphorylation at the murine synapse. Mol. Cell Proteomics. 11, 215-229 (2012)

19. $\mathrm{Xu}, \mathrm{S}$. et al. Proteomic analysis reveals O-GIcNAc modification on proteins with key regulatory functions in Arabidopsis. Proc. Natl Acad. Sci. USA 114, e1536-e1543 (2017).

20. Lin, C. et al. AFF4, a component of the ELL/P-TEFb elongation complex and a shared subunit of MLL chimeras, can link transcription elongation to leukemia. Mol. Cell. 37, 429-437 (2010).

21. Bartkowiak, B. et al. CDK12 is a transcription elongation-associated CTD kinase the metazoan ortholog of yeast Ctk1. Gene Dev. 24, 2303-2316 (2010).

22. Linker, K. et al. Involvement of KSRP in the post-transcriptional regulation of human iNOS expression-complex interplay of KSRP with TTP and HuR. Nucleic. Acids. Res. 33, 4813-4827 (2005).

23. Blencowe, B. J., Issner, R., Nickerson, J. A. \& Sharp, P. A. A coactivator of pre-mRNA splicing. Gene Dev 12, 996-1009 (1998).

24. Maruyama, T. et al. Roquin-2 promotes ubiquitin-mediated degradation of ASK1 to regulate stress responses. Sci. Signal. https://doi.org/10.1126/SciSignal.2004822 (2014).

25. Bhattacharyya, D. \& Glick, B. S. Two mammalian Sec16 homologues have nonredundant functions in endoplasmic reticulum (ER) export and transitional ER organization. Mol. Biol. Cell. 18, 839-849 (2007).
26. Ku, N. O. \& Omary, M. B. Identification of the major physiologic phosphorylation site of human keratin 18: potential kinases and a role in filament reorganization. J. Cell Biol. 127, 161-171 (1994).

27. Peng, J. et al. Effect of NPM1 type B mutation on the proliferation, invasion and chemosensitivity of THP-1 leukemia cells. Pharmazie. 72, 608-613 (2017).

28. Kim, K. \& Yeo, S. Extracellular chaperonin 10 augments apoptotic cell death induced by 5-fluorouracil in human colon cancer cells. Tumori. 100, e230-e235 (2014).

29. Liang, H. H. et al. Heat shock protein 27 influences the anti-cancer effect of curcumin in colon cancer cells through ROS production and autophagy activation. Life Sci. 15, 43-51 (2018).

30. Li, J., Tang, C., Li, L., Li, R. \& Fan, Y. Quercetin blocks t-AUCB-induced autophagy by Hsp27 and Atg7 inhibition in glioblastoma cells in vitro. J. Neurooncol. 129, 39-45 (2016).

31. Liang, C., Xu, Y., Ge, H., Li, G., \& Wu, J. The clinicopathological and prognostic value of HSP27 in hepatocellular carcinoma: a systematic review and metaanalysis. Onco. Targets Ther. https://doi.org/10.2147/OTT.S154227 (2018).

32. Sheng, B. et al. Increased HSP27 correlates with malignant biological behavior of non-small cell lung cancer and predicts patient's survival. Sci. Rep. https://doi.org/ 10.1038/s41598-017-13956-2 (2017).

33. Netsirisawan, P. et al. Decreasing O-GlcNAcylation affects the malignant transformation of MCF-7 cells via Hsp27 expression and its O-GIcNAc modification. Oncol. Rep. 40, 2193-2205 (2018).

34. Zahari, M. S. et al. Phosphoproteomic profiling of tumor tissues identifies HSP27 Ser82 phosphorylation as a robust marker of early ischemia. Sci. Rep. 5, 13660-13660 (2015).

35. Stetler, R. A. et al. Phosphorylation of HSP27 by protein kinase D is essential for mediating neuroprotection against ischemic neuronal injury. J. Neurosci. 32 2667-2682 (2012).

36. Robinson, A., Dunn, M. J., Mccormack, A., Remedios, C. D. \& Rose, M. L. Protective effect of phosphorylated $\mathrm{Hsp} 27$ in coronary arteries through actin stabilization. J. Mol. Cell Cardiol. 49, 370-379 (2010).

37. Haroshdavidovich, S. B. \& Khalaila, I. O-GlcNAcylation affects $\beta$-catenin and Ecadherin expression, cell motility and tumorigenicity of colorectal cancer. Exp. Cell Res. 364, 42-49 (2018).

38. Chen, Y. et al. NRAGE induces $\beta$-catenin/Arm O-GlcNAcylation and negatively regulates Wnt signaling. Biochem. Biophys. Res. Commun. 487, 433-437 (2017).

39. Du, C., Jaggi, M., Zhang, C. \& Balaji, K. C. Protein kinase D1-mediated phosphorylation and subcellular localization of beta-catenin. Cancer Res. 69 1117-1124 (2009).

40. Van Veelen, W. et al. $\beta$-catenin tyrosine 654 phosphorylation increases Wnt signalling and intestinal tumorigenesis. Gut. 60, 1204-1212 (2011).

41. Olsvik, H. et al. Endosomal microautophagy is an integrated part of the autophagic response to amino acid starvation. Autophagy. 15, 182-183 (2019).

42. Namkoong, S., Cho, C. S., Semple, I. \& Lee, J. H. Autophagy dysregulation and obesity-associated pathologies. Mol. Cells. 41, 3-10 (2018).

43. Wild, P. et al. Phosphorylation of the autophagy receptor optineurin restricts salmonella growth. Science. 333, 228-233 (2011).

44. Hasan, K. et al. CARF Is a vital dual regulator of cellular senescence and apoptosis. J. Biol. Chem. 284, 1664-1672 (2009).

45. Hasan, K. et al. Functional polymorphism of the CK2alpha intronless gene plays oncogenic roles in lung cancer. PLoS ONE 5, e11418 (2010).

46. Xiao, J., Liu, C., Chen, P. L. \& Lee, W. RINT-1, a novel Rad50-interacting protein participates in radiation-induced G2/M checkpoint control. J. Biol. Chem. 276 6105-6111 (2001).

47. Yang, Q. et al. NRAGE promotes cell proliferation by stabilizing PCNA in ubiquitin-proteasome pathway in esophageal carcinomas. Carcinogenesis. 35, 1643-1651 (2014).

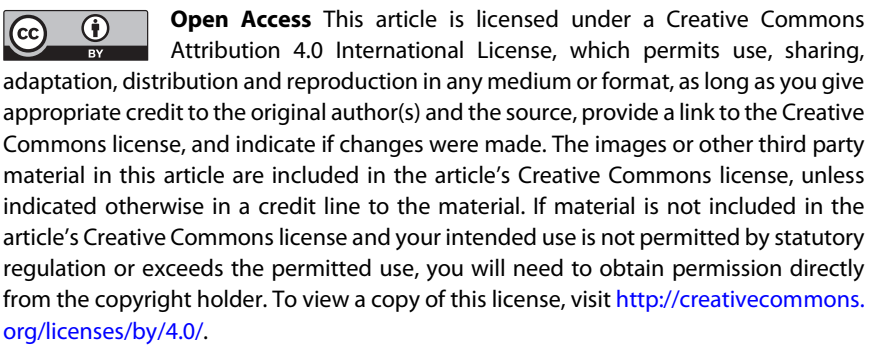

C) The Author(s) 2019 